\title{
Trauma, Research, and EMDR: A Disaster Responder's Wish List
}

\author{
Robert A. Gelbach \\ EMDR Humanitarian Assistance Programs
}

\begin{abstract}
Disasters, both natural and "man-made," affect a large portion of the Earth's population and can be expected to increase in intensity over the coming decades. The impact of disasters on mental health of affected populations is substantial and likely to be insufficiently addressed in the overall context of disaster response. While successful mental health intervention has been demonstrated in a variety of cases, including through the use of EMDR treatment, this problem needs more attention. Effective mental health response will be greatly supported by increased research on questions related to the incidence, form, and prognosis of disaster-generated traumatic stress, as these are affected by type of disaster, culture of affected population, sociological conditions, and neuropsychological factors, and the interactions among these. A brief summary of desirable research is presented that could help responders meet these challenges.
\end{abstract}

Keywords: disaster; PTSD; trauma; EMDR; research; disaster-response

$\mathbf{T}$ he pattern and impact of community-wide disasters throughout the world are changing. Our understanding of psychological traumatization and its treatment is also developing rapidly. Efforts by governments, nongovernmental organizations, and local communities to recover from disaster are affected both positively and negatively by these interconnected developments. Within the world of disaster response, mental health response is a small and underdeveloped corner (Satcher, Friel, \& Bell, 2007).

The EMDR Humanitarian Assistance Program (HAP) is among many operating in that corner. HAP has learned to address and accommodate the principles of good disaster response practice throughout its 13-year history. HAP has sent EMDR clinicians to postdisaster environments to provide brief treatment for survivors and first responders and to teach EMDR to local mental health professionals. HAP projects have occurred on five continents and several dozen countries, responding to earthquakes, hurricanes, and floods, as well as the aftermath of terrorism and low intensity warfare (HAP, 2008). Along the way HAP volunteers have become aware of circumstances that are not well addressed by research and have confronted circumstances that may throw some of the received wisdom about disaster response into question.

To be sure, a growing body of studies casts important light on incidence, consequence, and efficacy of response to mass trauma. An extensive database exists (PILOTS, 2008), and a wealth of good advice and reviews of past efforts can also be found in recent surveys (Norris, Galea, Friedman, \& Watson, 2006). The thrust of this article is to encourage rapid expansion of these investigations and to guide interventions and support policymakers who will determine the direction and extent of mental health interventions for disaster survivors.

This article concludes with a wish list for further research-composed in light of HAP's experience with disaster and EMDR on five continents. From the beginning, clinicians have found EMDR to be an effective psychotherapy for survivors of community-wide disasters who present with posttraumatic stress disorder (PTSD) (Grainger, Levin, Allen-Byrd, Doctor, \& 
Lee, 1997). EMDR has been effective across cultures and in response to stressors that vary widely in their nature, from natural disasters like earthquakes, floods, and storms to events arising in military combat or among civilians caught in political violence and other forms of continuing community-wide traumatic stress (Gelbach \& Davis, 2007).

\section{Disaster as a Phenomenon}

Operationally, disaster is an overwhelming misfortune that is not easily overcome or set right. Private disasters befall individuals or small groups, such as a family unit. Community disasters radically disturb and disrupt the context in which a whole community lives its life. The disaster may be a physical event or process, like an earthquake, or a social upheaval, like a war or revolution in a valued order. It may occur quickly or persist over time, like a drought or extended conflict. When whole communities, regions, or nations are disrupted and overwhelmed, the fabric of normal life for great numbers of people is simultaneously shredded so that they simply cannot go on with business as usual, but they must find a way to cope with adversity and retrieve what they can of their past life, wellbeing, and future hopes. The shared nature of their misfortune is a critical component of the disaster: It affects them as individuals and as members of society, a status that is often mediated by their more intimate membership within a family system (Gelbach $\&$ Davis, 2007).

Four aspects of disaster will be addressed in the following sections.

- First, it is likely that major disasters will befall large human communities with increasing frequency and intensity in the next several decades.

- Second, disasters generate neurophysiological trauma disorders, and we need to understand more about how, when, and why this occurs and to whom.

- Third, untreated trauma disorders can, in turn, extend and regenerate disasters, in ways and degrees that we need to better understand.

- Finally, we need to consider the range of treatment options for disaster-generated trauma. Specifically, we need to formulate strategies for trauma treatment that continuously weigh costs and benefits, since advances in our understanding of trauma and therapy are continuously interacting with the changing patterns of disaster to shift costs and benefits. With focused research and an open mind, disaster responders can keep ahead of these changes and maximize their effectiveness.

\section{Disasters Will Increase in Coming Decades}

While disaster is a break with normal life, it is far from an unusual state of affairs. Wherever we look, disasters are rising in frequency and intensity. The so-called "postwar" world of the later 20th century was in fact a period of nearly continuous warfare around the globe, frequently fought in small countries by armies that were proxies for great powers. The breakup of former empires, from the Ottomans through the Soviets, generated unstable nation-states that have frequently wavered or failed as keepers of peace and promoters of civil progress. None of these trends shows any sign of letting up in the future.

The forms of intergroup conflict have also worsened in recent years. Highly destructive weapons systems have proliferated beyond the societies that developed them, and inhibitions against targeting civilians are continuously eroding between and within nations, along with fragile standards of international law that were once expected to restrain the use of force. Terror has been an instrument of both state and nonstate actors.

Meanwhile, massive natural disasters occur with striking frequency around the world, though only a handful of these events achieve the public awareness visited upon the South Asian tsunami of late 2004 or Hurricane Katrina. More than 400 natural disasters occurred worldwide in 2005, compared with fewer than 100 in 1975 (Emergency Disasters Database, 2007). The human scale of these natural disasters has also been growing and will continue to grow.

The true character of these disasters is poorly understood if they are categorized as simply "natural." In the first place, the rapid and long-term rate of human population growth has made most societies far more vulnerable to natural disaster. The human population doubled to 6.5 billion between 1960 and 2005, and a "midrange" estimate projects 9 billion humans by 2050 , but only if fertility declines more than $10 \%$ (Population Division, 2005). Ecologists are preoccupied with whether human communities, let alone other species, will be able to cope with the resulting environmental pressures. Floods, earthquakes, storms, and droughts do not affect more square miles of territory, but far more human beings are located in harm's way. Moreover, the zone of greatest geophysical instability on the Earth's surface is in societies with lower per capita economic and technological resources to withstand disaster or recover from it.

Recently, geophysicists investigating prospects for future undersea earthquakes and tsunamis off the 
coast of Indonesia identified the Sumatran city of Padang (population 825,000) as an inevitable site of future devastation. While they could not predict the likely year of such a disaster, they hoped it would be soon, since the city's population is growing very rapidly and the later the event the greater the human devastation would be. In effect, the scientists viewed as implausible the possibility that Indonesians would limit the growth of the city or relocate it out of harm's way or, even less likely, bring a halt to rapid population growth (Mydans, 2007). Very similar patterns of denial and delay regarding warnings of impending environmental devastation preceded Hurricane Katrina over many years in the region around New Orleans (Tidwell, 2006).

Long-term interlocking trends of population growth, climate change, and other environmental changes have additional threatening implications for human societies. Croplands are receding; desertification is spreading; rising ocean levels place the shoreline, where a major fraction of human communities are located, at risk for storm damage and long-term flooding. Environmental pollution is accelerating, especially in countries with rapidly growing populations and intense pressures to industrialize rapidly. Demand for energy, food, water, and manufactured goods progressively outgrows supplies, giving rise to pressures on different populations from outsiders who want to move in. Infectious agents spread more quickly to the most vulnerable societies on modern transportation vectors (World Watch Institute, 2000-2006).

Significantly, none of these threatening prospects is today a focus of a concerted effort that is deemed likely to eliminate or blunt the threat. By default, the "strategy" of humanity has become essentially to hope for the best and clean up any resulting mess however we can. The more likely result will be increasing intergroup competition to gain control of resources and regions of safety at the expense of others. Darfur has been cited as a case in point (Flannery, 2005). Competition for natural resources also motivates much of the current foreign policies of the United States, China, and the nations of the Middle East (Klare, 2001).

Such a stance puts great reliance and responsibility on those who respond to disasters. Enormous resources will be needed just to recover from what might have been mitigated or avoided in the first place. It is a task that will be simply impossible unless responders shift radically from reactive support after a disaster to a proactive role.

It is easy to imagine how proactive response might work in dealing with physical infrastructure needs or provision of food supply, emergency shelter, medical care, and subsequent rebuilding. Responders will need to know which parts of the world are most vulnerable to which likely disasters. They will need policies and a plan-before the fact-for first response and for later rebuilding, and they will need material resources and skilled personnel available when the disaster occurs.

These notions, though difficult to implement, are plausible enough with respect to meeting material needs - even though they were so poorly realized in the wake of Katrina or the tsunami. There is not yet a comparable standard of proactive disaster response in the realm of mental health. This seems to be true in several respects. In general, although mental disorders account for $12 \%$ of the global disease burden, $40 \%$ of countries have no mental health policy, and mental health expenditures account for less than $5 \%$ of total health expenditures in the majority of countries (Murthy, Bertolote, \& Epping-Jordan, 2001). Looking at mental health services in relation to disaster, deficits of reliable knowledge limit efforts to become more effective and responsive. In efforts to build mental health capacity in areas of the world most vulnerable to disaster, we need to know more about:

- What sort of traumatic responses are most likely to emerge from different kinds and locations of disasters.

- What kinds or mixtures of mental health intervention will reduce trauma symptoms and be well tolerated.

- What costs and benefits are likely to arise by reducing — or not reducing — psychological traumatization at the societal level after disaster.

There is now general consensus among investigators that PTSD is a universal phenomenon, though specific expression of symptoms may be culture bound (Marsella, Friedman, Gerrity, \& Scurfield, 2001). In particular regions, we need predictions of the scale and form of likely future disasters, and longitudinal estimates, based on past studies, of how these developments will be absorbed psychologically by different population sectors. Similarly, we could benefit from reviews of past studies to anticipate how prevailing cultures are likely to structure survivors' traumatic stress and behavioral response.

We also need greater attention to whether and how different types of disaster affect the incidence, form, and severity of traumatic stress. For example, what sorts of experiences in modern warfare generate what sorts of trauma among combatants or noncombatants? Is the range of traumas that arise from natural disasters, like earthquakes or floods, systematically different from traumas that arise from technological 
disasters, from bombing or from more personal encounters with violent actors? How do populations adapt to chronic traumatizing conditions, like persistent low-intensity warfare or extended famine, on a societal level? Do differences, if any, vary across cultures (Giacaman, Abu-Rmeileh, Husseini, Saab, \& Boyce, 2007)? Is past trauma history of individuals, or the developmental stage at which an individual was previously traumatized, a more important determinant of new traumatization than any of these considerations? And so on. Studies that have been done to date are suggestive but few, and they are narrow in scope.

Field experience of mental health responders has limited light to throw on these questions, though there are tantalizing clues. In several disaster situations (Turkey, Sri Lanka, Indonesia, Louisiana) HAP volunteers reported that the family nexus was critical for traumatized survivors. Sometimes, older family issues were retriggered, but often the personal meaning of the disaster was the stress it placed on the client's capacity to rely on or fulfill responsibilities to family members at critical moments in the disaster and its aftermath. In the case of combat PTSD, EMDR clinicians trained by HAP report that a high proportion of cases appears to arise among younger soldiers with past histories of childhood traumatization and that combat situations frequently trigger those earlier unprocessed trauma memories (HAP volunteers, 2005-2007).

Proactive response to disaster mental health needs will require an expansion of studies into patterns of traumatic presentation and forms and levels of traumatization likely to arise in different cultures and disaster contexts. These studies would essentially look at the social/cultural ecology of trauma in the wake of disaster.

\section{Disasters Generate Traumatic Stress Disorders}

There is also need to better understand trauma as a neurophysiological phenomenon. Many studies have documented that community disasters produce stress disorders (Norris \& Elrod, 2006). However, there is considerable variation and relatively little systematic comparative data in the claims about how much or what sort of disorder to expect. Two comprehensive reviews of existing studies of disaster and mental health reported that PTSD was observed in $74 \%$ of the studies and was probably the most debilitating condition, followed by depression, anxiety, and nonspecific distress (Murthy et al., 2001; U.S. Department of
Health and Human Services, 1999). In another review of studies between 1980 and 2003, PTSD in the first year postdisaster ranged from $30 \%$ to $40 \%$ for those "directly exposed," $10 \%$ to $20 \%$ for rescue workers, and $5 \%$ to $10 \%$ for the "general population" (Galea, Nandi, \& Vlahov, 2005).

What is typically reported in a major disaster is widespread stress disorder in the affected population. Over time the number of people with acute stress decline and a rising number of people meet the formal diagnosis of PTSD. Ultimately, there are reports of large numbers of people who are "not traumatized, just depressed" or "just anxious," or there is "a rising incidence of addiction" or "increased violence and uncontrollable anger," or "increased somatic complaints" and so on. There is a good deal of not-very-systematic counting of symptoms and frustrating efforts to compare data from different kinds of disasters across different cultures and in the wake of different levels and kinds of treatment.

Digging deeper into the phenomenon of trauma, we need to wonder how differences in environmental variables influence the amount or type of traumatization. Traumatic stress disorder is defined diagnostically in functional and behavioral termsthat is, in terms of symptoms and with substantial leeway for different modes of presentation. Which behavioral symptoms are more likely to prevail? Do these tendencies arise randomly? How much of the behavioral expression of trauma disorder is a consequence of particular intrapsychic or neurophysiological issues, and how much is shaped by the subject's culture and societal circumstances? And what implications do the answers have for treatment and for prognosis?

In the field, postdisaster, there is often a high degree of early stress response that does not meet the diagnosis for PTSD. Acute stress disorder (ASD) has its own character, and its relations to PTSD need to be better understood. How often does it decline rapidly without treatment; does it become a precursor of PTSD if not treated?

In Thailand, there were reports of hundreds of survivors hospitalized for psychosis immediately after the tsunami of 2004 who were probably experiencing ASD, a condition that psychiatrists have been treating briefly and effectively in Israel with a variant of EMDR (Quinn, n.d.). In a study of Israeli civilians exposed to attacks during the Israel-Lebanon war of 2006, ASD affected a small proportion of those studied. Untreated, ASD accounted for the bulk of ultimate PTSD cases. When persons with ASD were given brief and early EMDR treatment, subsequent PTSD incidence was 
minimal (Kutz, 2007; Silver, Rogers, Knipe, \& Colelli, 2005).

There are also traumatic stress responses that do not fully meet the PTSD diagnosis but are nonetheless distressing and may be the modes of presentation for underlying PTSD. Depression and anxiety are examples of mental health diagnoses given to large groups, such as children on the Gulf Coast after Katrina. Clinicians who have treated some of these children with standard EMDR therapy have reported resolution of symptoms and have concluded that PTSD underlies the presenting symptoms (HAP, 2005-2007). We need formal studies that clarify these relationships. As long as the diagnosis of PTSD is based on constellations of symptoms rather than on underlying neurophysiological phenomena, the possibility exists that relevant therapy is underutilized for individuals who do not meet the conventionally defined diagnosis in its entirety.

Finally, many clinicians and disaster response planners claim that this or that group is naturally resilient and will do well or will bounce back after a disaster. This is a comforting belief if you do not know how to provide effective treatment to the group in question or if your treatment resources are scarce and you are looking for a task to put on hold. Resilience, or the capacity of a person to undergo stress and respond adaptively, is a very important capacity of all human beings. When individuals are overwhelmed by trauma, that simply means their resilience has been exceeded by a stressor.

To what extent does an individual's resilience depend on genetic or deeply rooted learned responses or culture? To what extent is it compromised by environmental circumstances, such as poverty or isolation, or increased with age and maturity or by specific training? How and when can we proactively use our knowledge of resilience to limit or treat mass traumatization? And when must we reject arguments that this or that group will be fine without therapy because they are "naturally resilient," as some mental health leaders in Louisiana asserted early on in New Orleans about children after Katrina (HAP, 2005-2007).

On the other hand, we have reports of children living in a setting of ongoing traumatic stress - a Palestinian refugee camp near Bethlehem-who were diagnosed with multiple symptoms of PTSD after soldiers fired on their rooftop play area, wounding one child. After two sessions of EMDR group treatment, a subsequent and similar traumatic event occurred. The local clinicians were surprised to find that the children rebounded very quickly after their third EMDR treatment, suggesting that prior trauma therapy had im- parted the potential for quicker recovery from future trauma, with appropriate therapy (Zaghrout-Hodali, Alissa, \& Dodgson, this issue).

It would help if we had better notions of who is likely to be resilient and how resilience might be increased before as well as after a disaster. In particular, for EMDR clinicians who have helped clients prepare for futures that may involve a re-encounter with stimuli that were once triggers, it would be interesting to know if therapeutic attention to future hopes, fears, and expectations can function as a method for building measurable resilience.

In summary, becoming proactive in mental health response to disaster involves having better tools to forecast where traumatization will occur, to what extent, and in what forms. It involves increased knowledge of why some people are traumatized in a disaster and others are not-knowledge that may explore links between trauma and genetics, trauma and individual life experiences and learning, trauma and broader sociocultural norms and patterns of meaning.

\section{Trauma Extends and Engenders Disaster}

Because trauma disorders do not affect most disaster survivors and are usually not life-threatening, and because acute stress disorders often abate over time, those who set overall disaster response policies tend to assign a lower priority to mental health response than to obviously pressing issues such as physical safety, food supply, basic shelter, and acute medical care. That is not obviously unreasonable, but it would be helpful to have some studies that assess specifically the contribution that restoring safety and basic needs can make to limiting subsequent traumatic stress disorders.

A separate question is how well such environmental normalization supports the psychological stabilization that is a prerequisite for traumatized survivors to be available for therapy. As efforts are made to restore facilities and services disrupted by a disaster, which of these factors are most likely to also promote psychological stabilization, even in survivors who are symptomatic for PTSD? Many visitors to New Orleans soon after Katrina came away with a profound sense that the loss of housing and the inability to keep neighborhoods and even families together for lack of shelter was a major contributor to psychological destabilization (HAP, 2005-2007). But did anyone study this formally?

For other reasons, planners should not fail to appreciate the impact of untreated trauma on longer-term recovery of communities that experience disasters. 
Aside from humanitarian considerations of alleviating distress, when large-scale traumatization is untreated it can effectively remove or reduce the economic and social contribution of the affected population at the time when their most energetic efforts are needed for community recovery. Children and adolescents who are unable to focus on education; parents who are impaired in their parenting role or in making a livelihood; untreated trauma survivors who self-medicate with addictive substances or who fall into self-destructive behavioral patterns such as uncontrolled anger: these are not only problems for the individual; they limit the larger community's capacity to recover. In this sense, trauma disorders may extend the negative effects of a disaster. We need to know, through longitudinal studies, more about when, how, and to what extent these negative effects come about and how to intervene to prevent or reduce them.

Many communities have been affected by what we might call a chronic disaster-a long, unabated struggle to recover from a series of ongoing misfortunes. Such is the plight of communities of permanent refugees or African villages filled with AIDS orphans who need more highly functioning workers. In these places, untreated trauma may be an important obstacle to recovery. Does such a community make better use of economic and other development resources after substantial trauma treatment is provided when compared with the response of a similar community without treatment?

One frequently noted phenomenon in disasters is that the target memories arising in EMDR treatment often relate to traumas experienced years earlier in disaster survivors' individual lives. In effect, the disaster functions as a mass trigger of unresolved trauma memories within the affected community. To that extent, each time new traumas are visited upon the community and not treated, a reservoir of memories is created that may more readily be triggered by the next disaster, like a bolt of lightning striking a forest stuffed with increasingly flammable underbrush.

It is thus possible that untreated trauma can engender disaster. In many parts of the world a succession of community disasters has occurred over many years, even generations. Famines or persecutions are followed by wars or deadly intergroup conflicts; spasms of violence may break out episodically with devastating consequences. Levels of hostility, distrust, and aggression toward the "Other" become endemic and are manipulated by political elites or other powerful forces within the society. A shocking number of outright genocidesat least 26-have been conducted since 1900 (Diamond, 2006). Survivors of such events may carry unresolved traumas throughout their own lives and may pass on hostilities and fears to their own offspring along an intergenerational transmission belt of traumatic stress (Auerhahn \& Laub, 1998).

Do these hostilities represent adaptations to trauma that have become acculturated as dysfunctional "norms" of societal membership? While postdisaster order may be restored in communities by reasserting prior systems of control, norms, and meaning, those systems may themselves rest on recent or age-old hatreds, fears, and hostilities-what might count as mal-adaptive information processing (to paraphrase Shapiro) at the societal level (Shapiro, 2006). There are cycles of violence and destruction, but the participants cannot envision or work effectively toward new relations.

Is there a shared network of untreated trauma memories at work here? Can trauma treatment break such cycles of conflict after they are well established? Could early treatment of new trauma prevent the establishment of such cycles? These are big questions, but trauma research may well throw light on them. We do have occasional clinical reports of individuals who have processed traumatic memories arising from social conflicts and have made authentic personal connections with past enemies. On the other hand, studies of trauma histories among contemporary terrorists are rare.

In summary, proactive mental health response to disaster requires giving due weight to the possibility that untreated trauma after a disaster may create a societal storehouse of dangerous traumatic material waiting to be retriggered by the next major societal stressor. In societies that are likely to undergo repeated disasters, avoiding or tamping down this possibility may be very important.

\section{Continuously Reviewing Costs and Benefits of Trauma Treatment in Disaster Response}

Trauma response has costs as well as benefits. There are never enough resources to meet the human needs that arise in any large disaster. Disaster response is always a matter of triage and setting priorities, often with tragic but least-bad consequences. Attention to life-threatening conditions and stabilizing actions appropriately comes first in time. Mental health response needs to take its place in the overall effort to recover from disaster.

That said, however, disaster response officials are continuously in danger of underutilizing mental health resources, simply because the state of the art in trauma treatment and the state of the science in traumatology 
are advancing rapidly and it takes time and effort to absorb the practical implications. For that very reason, researchers and practitioners in trauma fields should make a special effort to monitor the needs of disaster responders and communicate new findings continuously to them in "plain language" formulations. Disaster response officials, in turn, need to continuously recalculate the costs and benefits of mental health interventions so that their practice reflects new knowledge.

Here are some considerations that might well figure in current assessment of costs and benefits.

\section{Connecting Psychosocial Education, Stabilization, and Treatment}

Most clinicians working on trauma response believe that psychoeducation about trauma and its symptoms can be effective after a disaster in calming overwhelmed people, reassuring them that they or their family members and neighbors are not "going crazy" and that they can get help with their symptoms. We need more investigation about what modes of psychoeducation are most effective. We also need to find out if general public education about trauma before a disaster can reduce traumatization of those taught, as well as reduce trauma among those they care for, such as children for whom an overwhelmed caregiver may itself be a source of traumatic stress. If it is effective as a preventive step, then early-even predisasterpsychoeducation may be much less costly than later treatment.

Similarly, good treatment requires appropriate attention to stabilization before and during attempts to process trauma memories. There are many methods of stabilization, and some of them are particularly comfortable to members of particular cultures. We need to know more about these compatibilities, as well as whether it would be desirable to teach stabilization techniques widely, as a preparatory step, before a disaster occurs.

In those countries where the great majority of the world's people live, and particularly in societies that are currently most vulnerable to disaster, there is typically a very low proportion of mental health clinicians to the general population. Disasters tend to overwhelm the clinical resources of the society. HAP has responded to this circumstance by promoting simultaneous training of clinicians in best-practice trauma therapy and of paraprofessional human service workers in traumatology and stabilization methods. Clinicians and paraprofessionals frequently have poorly developed relationships for collaboration, but if they can get beyond this, the far more numerous paraprofessionals can specialize in case finding, stabilization, and preparing clients for efficient referral to scarce clinicians, who will be able to spend their time on those tasks they alone can do.

In HAP projects in diverse cultural settings, EMDR has been readily integrated with prevailing modes of stabilization, which can be accomplished by paraprofessionals. When later stages of the EMDR protocol are utilized by clinicians, EMDR is culturally well accommodated because it works directly with both cognitive and noncognitive memories as they naturally arise in the culture of the client.

\section{Creating Capacity in Areas of Potential or Continuing Need}

Good working relationships among psychoeducators, teachers of stabilization methods, and clinicians can extend care very efficiently. But these relationships are not likely to develop in the wake of disaster. They must be built up systematically in advance. The need to strengthen indigenous mental health service capacity in all countries has been widely recognized in statements of principle, like the guidelines issued by the International Society for Traumatic Stress Studies, which also stress the need to coordinate services with governmental and health care agencies (Task Force, 2002). These are important principles, but there has not yet developed a widespread movement to apply them proactively. We need projects that attempt to build trauma treatment capacity and research that assesses the attempts to help us improve the results.

For HAP, these principles have inspired a fundamental shift from a policy of responding to disaster to a policy of helping community mental health leaders prepare for potential disaster while building up basic skills in trauma treatment. Ideally, such help is requested by an indigenous health authority or provider agency, which will integrate new methods of care into its services and move its country or community to a status of self-sustainability in its capacity to treat trauma and respond to disaster mental health needs. In recent years, HAP has assisted such developmental projects in Turkey, India, Palestine, and Indonesia (Konuk, Knipe, Eke, Yuksek, \& Yurtsever, 2006). More recently HAP has extended this work to Kenya, the Philippines, and Lebanon.

\section{Connecting Treatment and Community Recovery Approaches}

Some psychosocial interventions are specifically chosen to be nonpsychotherapeutic. Advocates for these 
approaches argue that postdisaster psychotherapy is a flawed response because it "pathologizes" survivors and their efforts to cope with challenging circumstances without providing resources and motivations to restore disrupted communities and societal support systems to effective functioning (Miller \& Rasco, 2004).

Studies reported by these advocates do demonstrate that communities and many individuals are often highly effective in recovering from disaster when they are deliberately assisted to mobilize existing community resources and skills. However, their inclination to dismiss clinical services as irrelevant is not convincingly supported. Varying and large numbers of people are indeed traumatized in a clinical sense by their experience during disasters. Many of them will achieve rapid resolution of symptoms with good trauma treatment, and until their trauma is treated they will likely have reduced capacity to benefit from or contribute to community support programs. Finding out how many and which survivors fall into these categories is another question for research.

Considering that an important objective of EMDR treatment is focusing on enabling valued activities that previously caused anxiety or distress, it is most likely that good trauma treatment will increase a client's ability to become active in restoring his community postdisaster. A useful development would be open-minded exploration of how best to coordinate the full panoply of psychoeducation, psychosocial reconstruction, stabilization, and clinical treatment through collaborative partnerships of local clinicians, social service workers, and community leaders. Disaster responders may actually be doing this in different parts of the world, but research has not yet focused on it as a promising model.

\section{Researching Efficacy and Effectiveness of Treatment Options}

Finally, and importantly, mental health responders need to identify those therapies that are most efficacious and effective for particular conditions and in particular settings. Many therapies have not been very effective in addressing the needs and adapting to the conditions that prevail in the wake of disasters. Some therapies are culturally at odds with the populations of countries where they have been proposed. Some initiatives have been challenged as ineffective or even harmful.

Experience to date with EMDR is encouraging. It has been well received by both clinicians and clients in diverse cultures and societies outside the West.
Reports of clinicians and some early research efforts attest to its efficacy (Konuk et al., 2006). A number of adaptations of EMDR have been developed and utilized with clinical reports of success, such as the use of the Recent Events protocol for relatively brief treatment of individuals and the use of group treatment protocols, particularly with children who have shared a traumatic experience (Fernandez, Gallinari, \& Lorenzetti, 2004; Jarero, Artigas, \& Hartung, 2006). Treatment guidelines have positively reviewed the efficacy and effectiveness of EMDR in various contexts, including the treatment guidelines for combat PTSD (Department of Veterans Affairs \& Department of Defense, 2004).

Nonetheless, there is great need for more research into efficacy and effectiveness of different therapies in diverse settings. But field conditions postdisaster often make randomized clinical trials impractical. Other methodologies are available, however (Gibson, Hamblen, Zvolensky, \& Vujanovic, 2006). Comparative assessments of different therapies are needed and would undoubtedly contribute to cost-effective choices (Jaberghaderi, Greenwald, Rubin, Dolatabadim, \& Zand, 2004). To date much that we think we know about such comparisons comes from metaanalyses that do not provide a head-to-head comparison in diverse settings. The more our understanding of how psychological and cultural factors shape specific forms of traumatization, the more we will need to continue to research the best ways to deploy therapy as a key element in mental health response to disaster.

\section{Summary: A Disaster Responder's Wish List}

Here are some wishes for future research on trauma and disaster, grouped in the categories discussed above:

1. The incidence of disaster is likely to increase in coming decades:

- Where will disasters of different types most likely occur, with what differential impact on particular segments of the population?

- How are systematic differences in culture, age, gender, or social stratum likely to affect the incidence, form, and severity of disaster trauma for individuals and communities?

- How can emergency response planners use this information to anticipate and prepare for disaster trauma?

2. Disaster-generated traumatic stress disorders are neurophysiological phenomena: 
- Why do different people respond with different symptoms when traumatized?

- Can understanding these differences guide us to better treatment or prevention strategies in mass trauma situations?

- Can a better understanding of the interactions between culture and neurophysiological trauma response improve treatment or prevention?

- Are other responses to traumatic stress (such as ASD, depression, or anxiety) part of the same neurophysiological spectrum as PTSD, classically defined in the DSM-IV; if so, what implications does that have for therapeutic interventions?

- What factors in individual psychology or culture impart or promote resilience?

- Can education about trauma and enhanced stabilization skills reduce susceptibility to subsequent traumatic stress?

- Can future resilience be promoted in trauma survivors through therapy?

3. Unresolved shared trauma can engender future disasters:

- Can a better understanding of the sources or expression or distribution of traumatic stress tell us how a population is likely to be disadvantaged in meeting future stresses?

- Could therapeutic services to previously stressed societies reduce the likelihood of trauma-induced cycles of intergroup conflict and violence?

4. Trauma interventions have costs as well as benefits:

- How can trauma treatment capacity be increased cost-effectively in areas of potential or continuing need?

- Can psychosocial education and stabilization by nonclinicians be integrated with clinical treatment to maximize efficacy and reduce costs?

- Is early clinical treatment of acute stress disorder a cost-effective way to reduce subsequent incidence of PTSD after disasters?

- How can the benefits of clinical treatment and of community recovery efforts be made mutually supportive rather than rivalrous for scarce disaster response resources?

- What are the current relative capacities of alternative therapies to process trauma memories and to be tolerated by disaster survivors?

\section{References}

Auerhahn, N. C., \& Laub, D. (1998). International memory of the Holocaust. In Y. Danieli (Ed.), International hand- book of multigenerational legacies of trauma (pp. 21-42). New York: Plenum Press.

Department of Veterans Affairs \& Department of Defense. (2004). VA/DoD clinical practice guideline for the management of post-traumatic stress. Washington, DC: Veterans Health Administration, Department of Veterans Affairs and Health Affairs, Department of Defense. Office of Quality and Performance publication 10Q-CPG/PTSD-04.

Diamond, J. (2006). The third chimpanzee. New York: Harper.

Emergency Disasters Database. (2007). The OFA/CRED international disaster database. Brussels, Belgium: University Catholique de Louvain. Retrieved March 21, 2008, from http://www.em-dat.net/ disasters/trends.htm

Fernandez, I., Gallinari, E., \& Lorenzetti, A. (2004). A school-based EMDR intervention for children who witnessed the Pirelli Building airplane crash in Milan, Italy. Journal of Brief Therapy, 2, 129-136.

Flannery, T. (2005). The weather makers. New York: Atlantic Monthly Press.

Galea, S., Nandi, A., \& Vlahov, D. (2005). The epidemiology of post-traumatic stress disorder after disaster. Epidemiologic Reviews, 27, 78-91.

Gelbach, R., \& Davis, K. (2007). Disaster response: EMDR and family systems therapy under communitywide stress. In F. Shapiro, F. W. Kaslow, \& L. Maxfield (Eds.), Handbook of EMDR and family therapy processes (pp. 387406). New York: Wiley.

Giacaman, R., Abu-Rmeileh, N. M. E., Husseini, A., Saab, H., \& Boyce, W. (2007). Humiliation: The invisible trauma of war for Palestinian youth. Public Health, 121(8), 563-571.

Gibson, L., Hamblen, J., Zvolensky, M., \& Vujanovic, A. (2006). Evidence-based treatments for traumatic stress: An overview of the research with an emphasis on disaster settings. In F. Norris, S. Galea, M. Friedman, \& P. Watson (Eds.), Methods for disaster mental health research (pp. 208-225). New York: Guilford Press.

Grainger, R. D., Levin, C., Allen-Byrd, L., Doctor, R. M., \& Lee, H. (1997). An empirical evaluation of eye movement desensitization and reprocessing (EMDR) with survivors of a natural catastrophe. Journal of Traumatic Stress, 10, 665-671.

HAP Volunteers. (2005-2007). Informal reports of EMDR Humanitarian Assistance Program volunteers in India, Sri Lanka, Thailand, the U.S. Gulf Coast, and trainers of military clinicians. Unpublished.

HAP. (2007). Research findings. Retrieved March 21, 2008, from http://www.emdrhap.org/emdr_info/researchand resources.php

Jaberghaderi, N., Greenwald, R., Rubin, A., Dolatabadim, S., $\&$ Zand, S. O. (2004). A comparison of CBT and EMDR for sexually abused Iranian girls. Clinical Psychology and Psychotherapy, 11, 358-368.

Jarero, I., Artigas, L., \& Hartung, J. (2006). EMDR integrative group treatment protocol: A post-disaster trauma intervention for children and adults. Traumatology, 12(2), 121. 
Klare, M. T. (2001). Resource wars: The new landscape of global conflict. New York: Henry Holt and Company.

Konuk, E., Knipe, J., Eke, I., Yuksek, H., \& Yurtsever, A. (2006). Effects of EMDR therapy on post-traumatic stress disorder in survivors of the 1999 Marmara, Turkey, earthquake. International Journal of Stress Management (in preparation).

Kutz, I. (2007). Presentation at EMDR Europe Conference, Paris, June 2007.

Marsella, A. J., Friedman, M. J., Gerrity, E. T., \& Scurfield, R. M. (Eds.). (2001). Ethnocultural aspects of posttraumatic stress disorder (pp. 529-538). Washington, DC: American Psychological Association.

Miller, K. E., \& Rasco, L. M. (2004). An ecological framework for addressing the mental health needs of refugee communities. In K. E. Miller \& L. M. Rasco (Eds.), The mental health of refugees (pp. 1-66). Mahwah, NJ: Lawrence Erlbaum Associates.

Murthy, R., Bertolote, J., \& Epping-Jordan, J. (2001). The World Health Report 2001: Mental health: New understanding, new hope. Retrieved November 11, 2007, from http://www.who.int/whr/2001/en/whr01_en.pdf

Mydans, S. (2007, July 21). Indonesian town begins preparation for the next tsunami. The New York Times. Retrieved March 21, 2008, from http://www.nytimes.com/2007/ $07 / 21 /$ world $/$ asia $/ 21$ indonesia.html?_r $=1 \&$ scp $=1 \&$ sq $=$ mydans + july $+21+2007 \&$ st $=$ nyt $\&$ oref $=$ slogin

Norris, F., \& Elrod, C. L. (2006). Psychosocial consequences of disaster: A review of past research. In F. Norris, S. Galea, M. Friedman, \& P. Watson (Eds.), Methods for disaster mental health research. New York: Guilford Press.

Norris, F., Galea, S., Friedman, M., \& Watson, P. (Eds.). (2006). Methods for disaster mental health research (pp. 20-42). New York: Guilford Press.

PILOTS Database. (2008). National Center on Post Traumatic Stress Disorder, Department of Veterans Affairs. Retrieved January 2008 from http://www.ncptsd.va.gov/ncmain/ publications/pilots/index.html
Population Division. (2005). Department of Economic and Social Affairs, United Nations Secretariat. Retrieved January 2008 from http://unstats.un.org/pop/dVariables/ DRetrieval.aspx

Quinn, G. (n.d.). Emergency room protocol. Reprinted in EMDR HAP military field manual. Hamden, CT: EMDR Humanitarian Assistance Program.

Satcher, D., Friel, S., \& Bell, R. (2007). Natural and man made disaster and mental health. Journal of the American Medical Association, 298, 21.

Shapiro, E. (2006). New notes on Adaptive Information Processing. Hamden, CT: EMDR Humanitarian Assistance Program.

Silver, S. M., Rogers, S., Knipe, J., \& Colelli, G. (2005). EMDR therapy following the $9 / 11$ terrorist attacks: A community-based intervention project in New York City. International Journal of Stress Management, 12, 29-42.

Task Force on International Trauma Training of the International Society For Traumatic Stress Studies. (2002). Guidelines for international training in mental health and psychosocial interventions for trauma exposed populations in clinical and community settings. Psychiatry, 65, 156-164.

Tidwell, M. (2006). The ravaging tide. New York: Free Press.

U.S. Department of Health and Human Services. (1999). Mental health: A Report of the Surgeon General. Rockville, MD: U.S. Department of Health and Human Services. Smoking Abuse and Mental Health Services Advisors; Center for Mental Health Services, National Institutes of Health, National Institute of Mental Health.

World Watch Institute. (annual issues, 2000-2006). State of the world. New York: W.W. Norton.

Correspondence regarding this article should be directed to Robert A. Gelbach, 64 Hall Street, Hamden, CT 06517. E-mail: rgelbach@emdrhap.org 